\title{
Medial Patellofemoral Ligament Reconstruction: A Longitudinal Study Comparison of 2 Techniques with 2 and 5-Years Follow-Up
}

\author{
Diego C. Astur ${ }^{*}, 1$, Gustavo B. Gouveia ${ }^{2}$, José H. de Souza Borges ${ }^{2}$, Nelson Astur ${ }^{3}$, \\ Gustavo G. Arliani ${ }^{1}$, Camila C. Kaleka ${ }^{3}$ and Moises Cohen ${ }^{4}$
}

\author{
${ }^{I}$ Centro de Traumatologia do Esporte do Departamento de Ortopedia e Traumatologia da Escola Paulista de Medicina/ \\ Universidade Federal de São Paulo, São Paulo, Brazil \\ ${ }^{2}$ Insituto Cohen, São Paulo, Brazil \\ ${ }^{3}$ Faculdade de Ciencias Médicas da Santa Casa de Misericórdia de São Paulo, São Paulo, Brazil \\ ${ }^{4}$ Departamento de Ortopedia e Traumatologia da Escola Paulista de Medicina/ Universidade Federal de São Paulo, \\ São Paulo, Brazil
}

\begin{abstract}
Background: The purpose of this study was to compare the results of two popular surgical techniques for medial patellofemoral ligament MPFL reconstruction with a minimum of two-year follow-up.

Methods: Fifty-eight patients with traumatic tear of the medial patellofemoral ligament were included in one of the two surgical groups. Group 1 MPFLs were reconstructed through graft endobutton fixation and Group 2 through graft anchor fixation into the patella. After two to five-year follow-up, patients were asked to answer knee function questionnaires (Fulkerson and Kujala) as well as the SF-36 life quality score.

Results: There were no statistical difference among postoperative Kujala, Fulkerson, and SF-36 questionnaires scores between Groups 1 and 2. There were statistical significant differences favorable to patients in Group 1 with a shorter follow-up length (2-5 years) compared to those with a longer period of 5-10 years for both Kujala and Fulkerson scores and no difference for group 2.

Conclusion: Both medial patellofemoral ligament reconstruction techniques had similar results in a two to ten-year follow-up according to functions and life quality questionnaires. Furthermore, endobutton fixation for the patellar edge of the graft had better results in patients with 2 years of follow-up than those with 5 years. Gender was not significant for surgical results. Moreover, group 1 patients had higher number of complications.
\end{abstract}

Keywords: Ligament reconstruction, medial patellofemoral ligament, MPFL, surgical technique.

\section{INTRODUCTION}

Nowadays, a higher number of medial patellofemoral ligament (MPFL) injuries are detected due to a growing number of knee traumatic events [1-3] and more accurate diagnostic imaging studies are available [4]. The MPFL, a primary stabilizer of the medial side of the patella $[5,6]$, is located in a layer under the vastus medialis oblique (VMO) muscle $[7,8]$. It has a femoral origin located proximally and posteriorly to the medial epicondyle and distal to the adductor tubercle while its patellar insertion covers the superior half of the medial border of the patella [7]. Its anatomical features make this ligament the main restrictor $(50$ to $60 \%$ ) of lateral subluxation or dislocation of the patella $[9$, $10]$.

*Address correspondence to this author at the Centro de Traumatologia do Esporte do Departamento de Ortopedia e Traumatologia da Escola Paulista de Medicina/ Universidade Federal de São Paulo, Av Pacaembu 1024, Pacaembu 01234-000 São Paulo, SP, Brazil; Tel: 5511 992139219;

E-mail: mcastur@yahoo.com
The current understanding of the anatomy of the MPFL and how to diagnose injury to this structure has led to an even more anatomical and functional surgical reconstruction technique [11-13]. Current reported techniques differ in graft options, ligament insertion site, and type of implant used for fixation (interference screw, anchors, endobutton) $[14,15]$.

Intra and post-surgical complications of the MPFL reconstruction generated numerous articles seeking the best way to obtain satisfactory results for this surgical treatment $[16,17]$.

Two of the most popular MPFL reconstruction techniques were: (1) graft fixation into the patella with an endobutton and femoral fixation with an interference screw $[18,19])$; and, (2) graft fixation into the patella with anchors and femoral fixation with an interference screw [20,21]. The purpose of this study is to compare the results of both surgical techniques for MPFL reconstruction with a minimum of two-year follow-up. 


\section{MATERIALS AND METHODS}

After institutional review board approval, a longitudinal study was conducted from December 2003 to June 2011. Inclusion criteria for this study consisted of (1) age between 18 and 45 years (skeletally mature patients); (2) traumatic tear of the MPFL diagnosed through a magnetic resonance imaging (MRI) study by an expert radiologist after a patellar dislocation history; (3) failure of nonsurgical treatment after at least 6 months of physical therapy rehabilitation. Patients with associated knee ligamentous injuries, patellar chondral injuries grade III or IV according to the International Cartilage Repair Society (ICRS) classification, additional patellar instability risk factors, such as trochlear dysplasia, malaligment as well as patella alta, and, injuries or previous surgical procedures within the same knee were not included. All patients that we had to do any additional procedures, such as lateral release, a tibial tuberosity transfer or a trochleaplasty were excluded.

After a prior sample size calculation, a total of 62 patients met inclusion criteria and were included in one of the two surgical groups. The patients were randomized through sealed envelopes. Group 1 MPFLs were reconstructed through graft endobutton fixation into the patella and Group 2 through graft anchor fixation into the patella. Both groups had interference screw fixation for the femoral insertion of the graft. Ligamentous graft was harvested from ipsilateral gracilis muscle tendon for all subjects. All patients had their surgeries performed by the same senior author of this study.

\section{Preoperative Evaluation}

Patients with a patellar dislocation history and knee pain were clinically evaluated and when a traumatic tear of MPFL was suspected, knee radiography was performed to analyze presence of a patellar tilt through Merchants' incidence, which can be quantified through the sulcus and congruence angles, and patellar height through a lateral film. Patellar height was quantified through the Insall-Salvati method [22], where both patellar tendon length and patellar greater axis are measured. Patients then underwent a computed tomography (CT) scan to analyze patellar and trochlear morphology, as well as patellar alignment through the tibialtubercle-trochlear-groove (TTTG) measurement, which is the distance between the tibial tubercle and the trochlear groove with a normal value in full knee extension between 10 and $15 \mathrm{~mm}[23,24]$.

Finally, an MRI of the knee was performed to diagnose and characterize the MPFL defect. After informed consent, if all inclusion criteria were met, patients underwent the surgical procedure described below.

\section{Surgical Technique}

Group 1 patients underwent ligament reconstruction through endobutton patellar fixation. Arthroscopic investigation was always performed before open surgical repair to detect associated injuries in the joint. Once this is completed, an anteromedial approach to the proximal third of the tibia is then carried out for gracilis muscle tendon graft harvesting. At this point, a medial parapatellar approach is performed layer-by-layer identifying the intersection of the superior and medial thirds of the medial border of the patella, where the anatomic insertion point is located. A transverse tunnel in the upper $1 / 3$ is then made in the patella under fluoroscopic guidance, initially with a guide wire followed by a number 6 patellar drill. The tendon graft is then passed through the tunnel guided by the wire and fixed with an endobutton. Next, the appropriate femoral fixation point is marked and confirmed by fluoroscopy. This location is typically $0.5-1 \mathrm{~cm}$ distal and anterior to the medial femoral epicondyle, between the epicondyle and the adductor tubercle. A guide wire is then passed followed by a number 6 drill. The femoral edge of the graft is also passed through the tunnel with guide wire assistance and tensioned and fixed with a number 7 x $28 \mathrm{~mm}$ interference screw with the knee in 30-45 degrees of flexion (Fig. 1). We conducted an isometric test to define the correct tension of the graft. For this, we engage the graft in the already fixed guide wire in the femur. We performed a flexion and extension of the knee and observe if there is a graft excursion. If this tour is less than $3 \mathrm{~mm}$ we considered that it is the proper tension for the fixation.

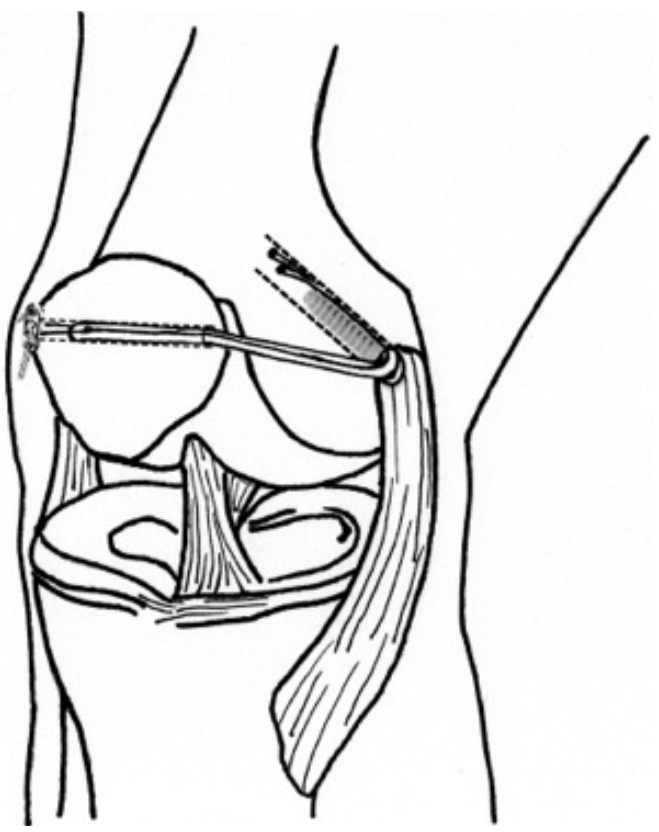

Fig. (1). Endobutton patellar fixation ilustration. A medial parapatellar approach is performed identifying the intersection of the superior and medial thirds of the medial border of the patella, where the anatomic insertion point is located. A transverse tunnel is then made in the patella and the tendon graft is then passed through the tunnel guided by the wire and fixed with an endobutton. The femoral edge of the graft is also passed through the tunnel and fixed with an interference screw with the knee in 30-45 degrees of flexion

Group 2 patients underwent ligament reconstruction through two metallic anchor fixation points on the patella. Diagnostic arthroscopy and graft harvesting are carried out in the same fashion as Group 1. A three $\mathrm{cm}$ medial parapatellar approach is performed. With the use of a small curette, a bony hollow is made at the superomedial border of the patella where two 3.2 absorbable anchors (ArthrexPushLock ${ }^{\circledR}$ ) are fixed and attached sutures are used to tie the patellar edge of the tendon graft into the patella. The femoral fixation point at the free end of the graft is marked and fixed 
with an interference screw in the same fashion as Group 1 (Fig. 2). There was no concurrent medial plication procedure performed in all ligamental reconstruction.

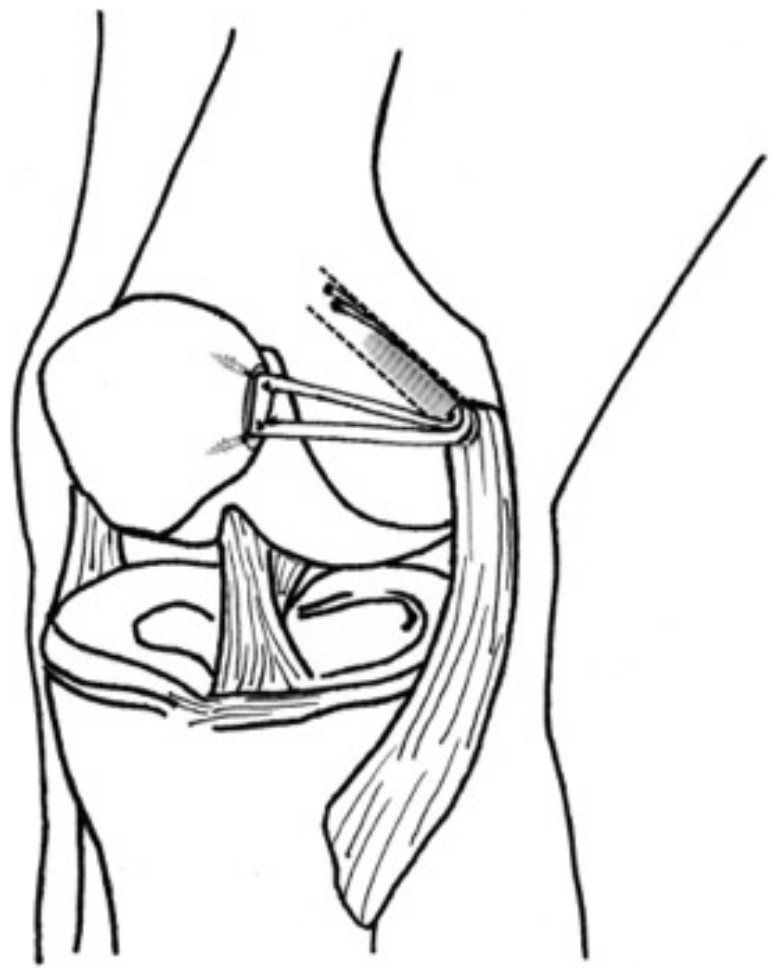

Fig. (2). Anchor fixation on the patella ilustration. A $3 \mathrm{~cm}$ medial parapatellar approach is performed and a bony hollow is made at the superomedial border of the patella where two anchors are fixed and attached sutures are used to tie the patellar edge of the tendon graft into the patella. The femoral fixation point at the free end of the graft is marked and fixed with an interference screw.

The graft was fixed with knee flexed between 30 to 45 degrees and enough tension to keep the graft with no laxity, as mentioned before.

\section{Post-Operative Treatment}

Within first two-postoperative weeks, physical therapy consisted of pain and swelling control as well as active assist range of motion without weight bearing. Quadriceps muscle strengthening started in the third postoperative week with partial weight bearing until fourth week. Full weight bearing was allowed from week four onward. Full activitiy including participation in sports was allowed 12 weeks after surgery.

We compiled data on basic patient characteristics (range of motion, patellofemoral pain, patellar stability), surgical details, and complications (restricted range of motion, recurrent lateral patellar stability, medial patellar instability, patellofemoral degenerative arthritis, and patellar fracture). Restricted range of motion was defined as an arthrofibrosis when the knee stiffness was less than 90 degrees and/or limited knee extension that negatively impacted patient function [25]. Follow-up of two years was required for the first post operative evaluation record. The second postoperative evaluation record occurred with a five-year follow-up. Out of the 62 patients, four were lost during follow-up and 58 completed the study. Out of the final 58 patients, 30 were included in Group 1 and 28 in Group 2.

After surgical ligamentous reconstruction, the medical staff (blinded fellows from the knee surgery program independent from the surgical team) clinically evaluated all patients every 15 days within the first month and then monthly until the sixth postoperative month. After at least two-year postoperative time point, patients were asked to answer knee function questionnaires such as the Fulkerson [26] and Kujala [27] as well as the SF-36 life quality score [28]. Two assessors of the outcome measures were blinded to the surgical procedure and clinical assessment evaluation. In addition, patellofemoral arthrosis was recorded through anteroposterior, lateral, and patellar axial radiographs views.

Questionaire results from Groups 1 and 2 were analyzed based on surgical technique and multivariate analysis was performed based on gender and length of follow up.

\section{Statistical Analysis}

Descriptive statistics were initially prepared followed by non-parametric testing with the Mann-Whitney test. SF-36, Kujala and Fulkerson item analysis consisted of Chi-square or Fisher tests. When necessary, class grouping was performed to meet appropriate frequency requirements in tables of contingency, determined by qualitative statistical tests [29]. The level of significance was set at $p<.05$. SPSS statistical package (version 20.0; SPSS, Inc., Chicago, IL, USA) was utilized.

\section{RESULTS}

Over the period, 58 patients were included (4 excluded by the necessity of additional procedures; 1 for Group 1 and 3 for Group 2), 30 in Group 1 and 28 in group 2. There were 28 females and 30 males. The mean age was 29.81 years (range 18-45). In-group 1, the mean age was 31,06 years (min 18- $\max 45$ years). In-group 2 , the mean age was 28,32 years ( $\min 18-\max 45$ years). There was no statistical difference between groups for age.

\section{Clinical Results}

Before and after surgery ( 2 and 5-years follow-up), pain and range of motion had a similar behavior in both groups. Visual analogue scale had an average of 7.3 points before surgery, 2.9 after two years and 4.1 after five years followup. On average, the range of motion before surgery was 73.5 degrees $(\mathrm{p}=0.65)$. After two years 128,4 degrees and 121,3 degrees after five years follow-up. $(\mathrm{p}=0,72)$ There is no difference between groups $(\mathrm{p}>0,05)$ (Table $\mathbf{1})$.

\section{Validate Scores Results}

\section{Postoperative Follow-Up Length}

When all patients from Groups 1 and 2 were analyzed together, shorter follow-up scores (two years) were significantly better than those with five years of follow-up according to Kujala $(p=0.009)$ and Fulkerson $(p=0.002)$. There was statistical significant difference favorable to patients in Group 1 with a shorter follow-up length (two 
years) compared to those with a longer period of five years for both Kujala $(p=0.009)$ and Fulkerson $(p=0.002)$ scores. (Table 2) Group 2 had no significant difference among patients with shorter and longer follow-up. No statistical significant difference for SF-36 score.

Table 1. Evaluation of all patients before surgery and after two and five years postsurgery. Groups 1 and 2 are similar about pain and range of motion evaluation.

\begin{tabular}{|c|c|c|c|}
\hline & Pre-Operative & $\begin{array}{c}\text { 2-Years } \\
\text { Follow-Up }\end{array}$ & $\begin{array}{c}\text { 5-Years } \\
\text { Follow-Up }\end{array}$ \\
\hline \multicolumn{4}{|l|}{ Pain (VAS) } \\
\hline Group 1 & 7.2 & 3.1 & 4.3 \\
\hline Group 2 & 7.4 & 2.7 & 3.9 \\
\hline All patients & 7.3 & 2.9 & 4.1 \\
\hline \multicolumn{4}{|c|}{ Range of Motion (Degrees) } \\
\hline Group 1 & $75.1^{\circ}$ & $126^{\circ}$ & $117.2^{\circ}$ \\
\hline Group 2 & $72.2^{\circ}$ & $131.4^{\circ}$ & $125.9^{\circ}$ \\
\hline All patients & $73.5^{\circ}$ & $128.4^{\circ}$ & $121.3^{\circ}$ \\
\hline
\end{tabular}

\section{Surgical Technique Results}

There was no statistical difference among postoperative Kujala, Fulkerson, and SF-36 questionnaires scores between Groups 1 and 2 in patients evaluated two and five years after surgery. There was a slight tendency for better surgical results in Group 1 technique (Table 3). However, statistical analysis showed significant difference on items of each questionnaire used that was favorable to Group 1 surgical technique when it comes to: Kujalas' limp $(p=0.001)$, support $(\mathrm{p}=0.050)$, and swelling $(\mathrm{p}=0.040)$; and Fulkersons' for pain $(\mathrm{p}=0.040)$.

\section{Gender}

There was no statistical difference in overall score among male and female patients in Group 1 and 2 even after two or five years postoperative.

\section{Complications}

There was one patient $(3 \%)$ treated with an endobutton (Group 1) who had a patella fracture before two years follow up analysis. Three patients (9\%) in Group 1 had subjective complaints of discomfort with the patellar endobutton positioning before two years follow up analysis too. There were also two cases that developed postoperative arthrofibrosis, one for each technique, during the fist six months of postoperative rehabilitation. The patella fracture was surgically treated with screw fixation and good result. The discomfort due to endobutton prominence was addressed with physical therapy and muscular strengthening. None was removed. Both patients who developed postoperative arthrofibrosis were surgically treated with a retinacular release, having good results although a small decrease in their knee range of motion was noted (Range of motion: 75 and 90 degrees before surgical release; 120 and 135 degrees after surgical release, respectively). No recurrent lateral patellar instability or medial patellar instability were observed.

\section{DISCUSSION}

Evaluation of both techniques showed no significant differences when compared to each other. A high number of surgical techniques and fixation methods led to numerous studies with the objective of comparing the most adequate surgical technique to anatomically reconstruct this structure, with lesser complication rates and better postoperative rehabilitation and life quality. Lenschow et al. compared 5 different techniques in a recent biomechanical study and concluded that transosseous fixation had similar results as fixations with an implant [30]. Gomes compared static reconstruction through the adductor magnus with dynamic reconstruction through semitendinosus tendon and concluded that even in the absence of statistical significance, the results suggest that a more dynamic femoral fixation can be more advantageous than a rigid alternative [31]. Kang et al. compared two techniques of anatomical MPFL reconstruction: primary femoral fixation and separate tightening of two bundles, and primary patellar fixation with simultaneous

Table 2. Comparison between time of follow-up - There was significant difference between patients evaluated 2 years after surgery and then evaluated 5 years after surgery when endobutton fixation was performed according to $K u j a l a s(p=0.009)$ and Fulkerson (

\begin{tabular}{|c|c|c|c|c|c|c|c|}
\hline & $\mathbf{N}$ & Average & SD & Median & Min & $\operatorname{Max}$ & p-Value \\
\hline \multicolumn{8}{|l|}{ Kujala } \\
\hline 2 years & 58 & 87.7 & 12.2 & 92 & 48 & 100 & $0.009^{*}$ \\
\hline 5 years & 58 & 79.6 & 14.5 & 83 & 29 & 96 & \\
\hline \multicolumn{8}{|l|}{ Fulkerson } \\
\hline 2 years & 58 & 94.1 & 8.4 & 95 & 58 & 100 & $0.002 *$ \\
\hline 5 years & 58 & 79.9 & 22.5 & 89 & 19 & 100 & \\
\hline \multicolumn{8}{|l|}{$S F-36$} \\
\hline 2 years & 58 & 130.1 & 16.0 & 136.0 & 78 & 143 & 0.387 \\
\hline 5 years & 58 & 131.0 & 11.8 & 135.0 & 84 & 143 & \\
\hline
\end{tabular}


Table 3. Comparison between groups - There was no significant difference between both techniques according to Kujala, Fulkerson and SF-36 scores. A slight tendency for significance was noted for Fulkerson total scores.

\begin{tabular}{|c|c|c|c|c|c|c|c|}
\hline & $\mathbf{N}$ & Average & SD & Median & Min & Max & p-Value \\
\hline \multicolumn{8}{|l|}{ Kujala } \\
\hline Group 1 & 30 & 82.1 & 14.4 & 84 & 29 & 100 & 0.116 \\
\hline Group 2 & 28 & 87.0 & 12.6 & 90 & 48 & 100 & \\
\hline \multicolumn{8}{|l|}{ Fulkerson } \\
\hline Group 1 & 30 & 83.7 & 21.0 & 91 & 19 & 100 & 0.060 \\
\hline Group 2 & 28 & 93.5 & 9.1 & 96 & 58 & 100 & \\
\hline \multicolumn{8}{|l|}{$S F-36$} \\
\hline Group 1 & 30 & 129.6 & 15.2 & 135.5 & 78 & 143 & 0.852 \\
\hline Group 2 & 28 & 131.6 & 13.3 & 135.0 & 83 & 143 & \\
\hline
\end{tabular}

tightening of the bundles. Conclusion was that separate tightening of bundles and primary femoral fixation had superior results [32]. However, the majority of comparative studies between techniques still has short follow-up with small number of patients and focus into the evaluation of the reconstructed ligament biomechanics. At the present study, 58 patients were gathered in the time period of 2003 to 2011 with a minimum follow-up of two years. Patients underwent current popular repair techniques although scarcely evaluated and compared in recent literature.

Main critic for our Group 1 reconstruction technique according to orthopaedic literature relates increasing in patellar bone fragility due to the tunnel drilled, which leads to a possible fracture during early postoperative period. Also, the endobutton becomes relatively shallow at the lateral border of the patella, generating discomfort to the patient. Besides that, the surgical technique used in group 1 uses only a single-bundle graft, whereas the technique used in group 2 uses a double-bundle graft. The surgical technique used in group 1 involves non-anatomical fixation of the graft, since the graft is fixed against the lateral border of the patella; the technique used in group 2 involves anatomical fixation of the graft, since the graft is fixed at the native attachment site of the MPFL on the medial border of the patella. In our study, one patient in Group 1 had a patellar fracture related to the technique while three patients complained of endobutton discomfort in their knees. Other than that, when Group 1 technique was compared to Group 2 , there was no significant difference between patients evaluated at least two years after surgery, but a slight tendency for better results with patellar anchor reconstruction was noted.

Patients evaluated were also divided into groups according to their follow-up time: two years and five years. Few are the studies with long term follow-up for MPFL reconstruction $[5,18,31,33]$, which makes the comparison of our results with previously reported data in the literature difficult. For patients with endobutton fixation (Group 1), shorter follow-up subjects had significantly better results than those with five years. When anchor fixation within the patella was performed (Group 2), there was no significant difference between longer and shorter follow-up, which is relevant, since those patients had no life quality or knee function decrease over time.

We also analyzed if gender had an impact over surgical results, but there was none significant, no matter which reconstruction technique was applied. However, female patients had better outcomes over males for some questionnaire items, such as squatting and physical functioning, which was statistically significant. No similar results were found in the literature. There is no set reason why the results found, but we believe it may be related to the musculoskeletal anatomy of women.

Study limitations include relatively small samples, although it is still one of the largest in the literature found for these specific reconstruction techniques. Furthermore, no preoperative score evaluation was quantified for pre and postoperative comparison. Finally, this study addresses only two techniques to surgically reconstruct the MPFL, although there are more than 20 different techniques previously reported in the literature.

\section{CONCLUSION}

Both medial patellofemoral ligament reconstruction techniques had similar results in a two and five-year followup according to Kujala, Fulkerson and SF-36 questionnaires. Furthermore, endobutton fixation for the patellar edge of the graft had better results in patients with two years of followup than those with five years. Gender was not significant for surgical results. Moreover, group 1 patients had higher number of complications.

\section{AUTHORS' CONTRIBUTIONS}

Diego Costa Astur: Corresponding author analyse the comparisons between groups and decide the study draw ( methodology).

Gustavo Borges Gouveia: Colect dates after the patients fill the questionaires.

Jose Humberto Borges: Colect dates after the patients fill the questionaires.

Nelson Astur: Text review and methodology. 
Gustavo Gonçalves Arliani: Colect dates after the patients fill the questionaires.

Camila Cohen kaleka: Colect dates after the patients fill the questionaires.

Moises Cohen: Surgeon and text review.

\section{CONFLICT OF INTEREST}

The authors confirm that this article content has no conflict of interest.

\section{ACKNOWLEDGEMENTS}

Declared none.

\section{REFERENCES}

[1] Howells NR, Barnett AJ, Ahearn N, Ansari A, Eldridge JD. Medial patellofemoral ligament reconstruction: a prospective outcome assessment of a large single centre series. J Bone Joint Surg Br 2012; 94(9): 1202-8.

[2] Sillanpää PJ, Salonen E, Pihlajamäki H, Mäenpää HM. Medial patellofemoral ligament avulsion injury at the patella: classification and clinical outcome. Knee Surg Sports Traumatol Arthrosc 2014; 22(10): 2414-8.

[3] Willis RB, Firth G. Traumatic patellar dislocation: loose bodies and the MPFL. J Pediatr Orthop 2012;32(Suppl 1): S47-51.

[4] Redfern J, Kamath G, Burks R. Anatomical confirmation of the use of radiographic landmarks in medial patellofemoral ligament reconstruction. Am J Sports Med 2010; 38(2): 293-7

[5] Burks RT, Desio SM, Bachus KN, Tyson L, Springer K. Biomechanical evaluation of lateral patellar dislocations. Am J Knee Surg 1998; 11(1): 24-31.

[6] Schöttle PB, Fucentese SF, Romero J. Clinical and radiological outcome of medial patellofemoral ligament reconstruction with a semitendinosus autograft for patella instability. Knee Surg Sports Traumatol Arthrosc 2005; 13(7): 516-21.

[7] Amis AA, Firer P, Mountney J, Senavongse W, Thomas NP. Anatomy and biomechanics of the medial patellofemoral ligament. Knee 2003; 10(3): 215-20.

[8] Aragão JA, Reis FP, de Vasconcelos DP, Feitosa VL, Nunes MA. Metric measurements and attachment levels of the medial patellofemoral ligament: an anatomical study in cadavers. Clinics (Sao Paulo) 2008; 63(4): 541-4.

[9] LeGrand AB, Greis PE, Dobbs RE, Burks RT. MPFL reconstruction. Sports Med Arthrosc Rev 2007; 15(2): $72-7$.

[10] Nomura E, Inoue M, Osada N. Anatomical analysis of the medial patellofemoral ligament of the knee, especially the femoral attachment. Knee Surg Sports Traumatol Arthrosc 2005; 13 (7): 510-5.

[11] Buckens CF, Saris DB. Reconstruction of the medial patellofemoral ligament for treatment of patellofemoral instability: a systematic review. Am J Sports Med 2010; 38(1): 181-8.

[12] Fisher B, Nyland J, Brand E, Curtin B. Medial patellofemoral ligament reconstruction for recurrent patellar dislocation: a systematic review including rehabilitation and return-to-sports efficacy. Arthroscopy 2010; 26(10): 1384-94.

[13] Singhal R, Rogers S, Charalambous CP. Double-bundle medial patellofemoral ligament reconstruction with hamstring tendon autograft and mediolateral patelar tunnel fixation: A meta- analysis of outcomes and complications. Bone Joint J 2013; 95-B (7): 9005 .

[14] Mikashima Y, Kimura M, Kobayashi Y, Asagumo H, Tomatsu T. Medial patellofemoral ligament reconstruction for recurrent patellar instability. Acta Orthop Belg 2004; 70(6): 545-50.

[15] Camanho GL, Bitar AC, Hernandez AJ, Olivi R. Medial patellofemoral ligament reconstruction: a novel technique using the patellar ligament. Arthroscopy 2007; 23 (1): 108-14.

[16] Shah JN, Howard JS, Flanigan DC, Brophy RH, Carey JL, Lattermann C. A systematic review of complications and failures associated with medial patellofemoral ligament reconstruction for recurrent patellar dislocation. Am J Sports Med 2012; 40(8): 191623.

[17] Tanaka MJ, Bollier MJ, Andrish JT, Fulkerson JP, Cosgarea AJ. Complications of medial patellofemoral ligament reconstruction: common technical erros and factors for sucess: AAOS exhibit selection. J Bone Joint Surg Am 2012; 94(12): e87.

[18] Drez D, Jr., Edwards TB, Williams CS. Results of medial patellofemoral ligament reconstruction in the treatment of patellar dislocation. Arthroscopy 2001; 17(3): 298-306.

[19] Nakagawa S, Arai Y, Kan H, et al. Medial patellofemoral ligament reconstruction procedure using a suspensory femoral fixation system. Arthrosc Tech 2013; 2(4): e491-5.

[20] Muneta T, Sekiya I, Tsuchiya M, Shinomiya K. A technique for reconstruction of the medial patellofemoral ligament. Clin Orthop Relat Res 1999; 359: 151-5.

[21] Farr J, Schepsis AA. Reconstruction of the medial patellofemoral ligament for recurrent patelar instability. J Knee Surg 2006; 19(4): 307-16.

[22] Insall J, Salvatti E. Patella junction in the normal knee joint. Radiology 1971; 101: 101-4.

[23] Caton JH, Dejour D. Tibial tubercle osteotomy in patello-femoral instability and in patellar height abnormality. Int Orthop 2010; 34(2): 305-9.

[24] Goutallier D, Bernageau J, Lecudonnec B. Mesure de l'écart tubérosité tibiale antérieure-gorge de la trochlée (TA.GT). Technique Résultats Intérêts. Rev Chir Orthop 1978; 64:423-8.

[25] Bonutti PM, Marulanda GA, McGrath MS, Mont MA, Zywiel MG. Static progressive stretch improves range of motion in arthrofibrosis following total knee arthroplasty. Knee Surg Sports Traumatol Arthrosc 2010; 18(2): 194-9.

[26] Fulkerson JP, Shea KP. Disorders of patelofemoral alignment. J Bone Joint Surg Am 1990; 72: 1424-9.

[27] Kujala UM, Jaakkola LH, Koskinen SK, Taimela S, Hurme M, Nelimarkka O. Scoring of patellofemoral disorders. Arthroscopy 1993; 9: 159-63.

[28] McHorney CA, Ware JE, Raczek AE. The MOS 36-item short form health survey (SF-36): II. Psychometric and clinical tests of validity in measuring physical and mental health constructs. Med Care 1993; 31: 247-63.

[29] Zar JH. Bioestatistical analysis. $5^{\text {th }}$ ed. Pratice-Hall/Pearson 2010.

[30] Lenschow S, Schliemann B, Gestring J, Herbort M, Schulze M, Kosters C. Medial patellofemoral ligament reconstruction: fixation strength of 5 different techniques for graft fixation at the patella Arthroscopy 2013; (12): 766-73.

[31] Gomes JE. Comparison between a static and a dynamics technique for medial patellofemoral ligament reconstruction. Arthroscopy 2008; 24(4): 430-5.

[32] Kang H, Cao J, Yu D, Zheng, Wang F. Comparison of different techniques reconstruction of the medial patellofemoral ligament: a prospective randomized study. Am J Sports Med 2013; 41(5): 1013-21.

[33] Nomura E, Inoue M, Kobayashi S. Long-term follow-up and knee osteoarthritis change after medial patellofemoral ligament reconstruction for recurrent patellar dislocation. Am J Sports Med 2007; 35(11): 1851-8. 